\title{
中国心理学前沿
}

\section{SNARC 效应的研究综述}

\author{
李章平* 何 华 晏婷婷 \\ 苏州大学心理学系, 苏州, 215325 \\ 邮箱: 154896923@qq.com
}

摘 要: 本文主要是在已有研究结果的基础上对 SNARC 效应进行综述和展望。 首先介绍了 SNARC 效应的起源和定义, 并总结了 SNARC 效应的特征和研究 方法; 接着对 SNARC 效应的本质和相关理论解释进行论述; 除此之外还结合 新近的认知神经科学的研究成果对 SNARC 效应的脑机制进行阐述; 最后提出 三个有待深入探讨的问题。

关键词: SNARC 效应; 数字表征; 注意转移; 顶叶皮层

收稿日期：2018-01-26; 录用日期：2018-04-15; 发表日期：2019-03-15

\section{The research overview of the SNARC effect}

\author{
Zhangping Li* Hua He Tingting Yan
}

Abstract: This paper mainly reviewed the SNARC effects based on the existing

文章引用: 李章平, 何华, 晏婷婷. SNARC 效应的研究综述 [J]. 中国心理学前沿, 2019, 1 ( 1) : 50- 
research results. Firstly, we introduced the origin and definition of the SNARC effect, and summarized its characteristics and research methods. Then we discussed the nature and theoretical explanation of SNARC effect. In addition, the brain mechanism of SNARC effect is described in combination with the recent research of cognitive neuroscience. Finally, three questions need to be further discussed.

Key words: SNARC effect; digital representation; attention transfer; the brain mechanism

Received:2018-01-26; Accepted :2018-04-15; Published:2019-03-15

Copyright (c) 2019 by author(s) and SciScan Publishing Limited.

This article is licensed under a Creative Commons Attribution-NonCommercial 4.0 International License.

https://creativecommons.org/licenses/by-nc/4.0/

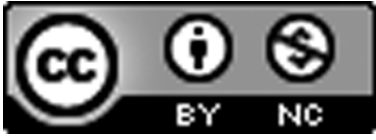

数字在我们的生活中有着非常广泛的应用，它不仅可以记录我们周围的空 间环境信息（如教室的长、宽、高），而且还是时间信息的载体（如当下的时 刻和我们的年龄都必须通过数字呈现出来），但我们却好像一直把数字看成是 一种理所当然的存在，直到 19 世纪后期，Galton 等发现了人们倾向于利用一些 空间特征来表达和描述数字的大小及其变化。例如，通过一条带箭头的直线来 表示数值的大小变化; 通过空间的距离来表示数值的大小差别以及通过用曲线 或折线来表示数值的变化趋势。Galton 的发现让心理学领域的学者看到了数字加 工和数字表征的研究价值, 并开启了数字认知领域研究的新时代。

SNARC 效应就从属于数字加工的研究领域。它是 Dehaene 等在研究数字与空 间的对应关系时发现的一种重要现象，他们通过精巧的实验设计揭示了数字大小 和反应键的空间位置之间存在一种对应关系，即位于身体左侧的反应键与对小数 
字的快速反应相联系，而位于身体右侧的反应键与对大数字的快速反应相联系， 他们把这种现象命名为空间一数字反应联和编码效应（Spatial-Numerical Association of Response Codes Effect, SNARC ) 。

\section{SNARC 效应的研究方法}

\section{1 大小比较任务}

数字大小比较任务是研究数字加工过程其空间特性自动激活的一个经典范 式。实验过程中要求被试对目标数字 $(1 \sim 9)$ 与标准数字 $(5)$ 进行比较, 如果 目标数字比 5 小，被试要用左手按左键来反应；如果目标数字比 5 大，被试要用 右手按右键来反应 $[1]$ 。该范式通常采用被试内设计，即每个被试完成两个实验 任务, 任务一中左手对小数字反应, 右手对大数字反应; 任务二要求反应手颠倒, 从而平衡左右手对刺激反应速度上的差异；而由实验的先后顺序引起的误差则在 被试间实现平衡。通过分析数字大小和反应位置之间的交互作用去探讨数字大小 和反应键的空间位置之间的对应关系，即数字大小和空间特性的关系。

\section{2 奇偶判断任务}

数字奇偶判断任务同样是 SNARC 效应的重要研究范式，与大小比较任务的不 同之处在于，奇偶判断任务中数字大小信息是与任务无关的，是研究 SNARC 效应 的一种隐蔽的方式，如果在该任务中发现 SNARC 效应的话就更加证明数字的空间 特性是自动激活的。实验过程中要求被试判断屏幕上呈现的数字是奇数还是偶数, 若是奇数，被试需要用左手按左键来反应；若是偶数，被试需要用右手按右键来 反应 [2]。和大小比较任务一样, 通常采用被试内设计来平衡左右手对刺激反应 速度上的差异，而由实验的先后顺序引起的误差则在被试间实现平衡。最终通过 分析数字奇偶和反应位置之间的交互作用对 SNARC 效应进行检验。

\section{3 自我报告任务}

自我报告任务是研究身体运动对 SNARC 效应影响的一个有效方法。该任务 
中通常设置三种条件：一种是头部或身体保持正直，作为基线水平条件; 另外两 种实验条件分别是头部或身体向左动和向右动（或向上动和向下动），要求被试 在每种条件下随机报告 $1 \sim 30$ 之间的任意数字，通过比较实验条件和控制条件 下被试报告小数字和大数字的频率的差异来探索低级的感觉运动对 SNARC 效应 的影响 [3]。除了用于研究主动的身体运动之外，研究者还利用这种范式探讨 被动的身体运动对 SNARC 效应的影响 [4]。

\section{4 目标探测任务}

目标探测任务多用于研究注意的 SNARC 效应或类 SNARC 效应, 这类效应是 一种由具有顺序信息的刺激材料 (如字母、月份等)所引起的空间注意转移的现象。 实验中通常先给被试呈现 1 个线索刺激，可以是数字，也可以是字母或者其他刺 激（如 $1 、 2 、 3 、 7 、 8 、 9$ 或 $\mathrm{a} 、 \mathrm{~b} 、 \mathrm{c} 、 \mathrm{x} 、 \mathrm{y} 、 \mathrm{z}$ ），接着被试的左右视野会各有一 个无意义方块，并在任意一个方块中呈现一个探测刺激。被试的任务就是对探测 刺激出现的位置做出按键反应，通过分析被试对左侧和右侧探测刺激的反应时间 与线索刺激的关系来检验 SNARC 效应或类 SNARC 效应的存在 $[5]$ 。

\section{SNARC 效应的特性}

\subsection{SNARC 效应的方向性}

水平方向的 SNARC 效应发现之后，研究者们认为应该还存在垂直方向的 SNARC 效应（大数位于上方，小数位于下方）和前后方向的 SNARC 效应（大 数位于前面, 小数位于后面）。这可能与我们的生活经历有关，例如我们在爬 楼梯的过程中，随着海拔的增高楼层数也在增大，下楼的时候随着海拔的降低 楼层数也在变小; 再比如说进步、将来都和时间轴上更远、更大的数字联系在 一起，而倒退、过去都和时间轴上更近、更小的数字联系在一起。垂直走向的 SNARC 效应已经在 Ito 和 Hartmann 等的研究中被证实, 他们选取日本人为被试, 要求被试对按上下排列的键对数字的奇偶性进行反应，结果和预期是一致的， 即确实存在垂直走向的 SNARC 效应 $[4][6]$; 此外 Chwarz 等采用眼动实验 
范式也同样发现了垂直方向上的 SNARC 效应 [7]。

但目前还没有实验的证据来证明从前往后方向的 SNARC 效应，究竟是假 设不成立, 还是实验设计不够合理, 这是值得我们思考的一个地方。基于此, 研究者们认为数字的空间表征更可能是一个两维的 “心理数字图”（ Mental Number Nap )。

\subsection{SNARC 效应的灵活性}

Dehaene 等通过一系列的实验发现 “心理数字线” 并不是僵化的，而是具有 很大程度的灵活性，人们可以根据当前实验任务的不同而对数字在心理数字线 上的表征进行动态的调整。他将之前的刺激序列分成 $0 \sim 5$ 和 $4 \sim 9$ 两种范围 再进行实验，结果发现，在 $0 \sim 5$ 系列中，4和 5 与右侧空间联系得更紧密一些， 而在 $4 \sim 9$ 系列中，4和 5 与左侧空间联系得更紧密一些; 此外王强强等 (2015) 的研究发现两种不同参考系下字母相对位置的颠倒能够引起字母加工中 SNARC 效应的反转, 这两项研究都说明数字引起的空间注意的转移依赖于其所处的数 字或字母背景及其所选择的参考系 $[2][8]$ 。

张宇、雷维娜和游旭群 (2010) 的研究从另一个角度证明了 SNARC 效应的 灵活性 [9]。他们采用刺激探测任务, 探讨负数的低水平加工能否引起空间注 意的转移, 结果发现, 如果呈现的线索全部都是负数的话就只引起了基于绝对 值的空间注意的转移，负号则变得没有意义；而如果把正数、负数和 0 一起作 为线索的话就引起了基于其数量值的空间注意的转移，说明此时被试把负数的 数字及其负号作为一个整体进行加工，同时也证明心理数字线可以延伸至 0 的 左边, 至于何时延伸至 0 的左边取决于组成这条心理数字线的所有数字 [8]。

\section{3 对经典 SNARC 效应的理论解释}

各类实验都证实 SNARC 效应是一种普遍的现象，但该如何解释这一现象 呢? 对此, 研究者们从信息加工的视角提出了各自的观点和理论模型, 其中包 括心理数字线理论、极性配对理论、双路线认知模型和计算理论模型。 


\section{1 儿理数字线理论}

Dehaene等于 1993 年最先提出人脑中存在一条从左向右逐渐递增的数字线, 小的数字被表征在线的左侧, 大的数字被表征在线的右侧, 并将其形象地称为“心 理数字线”（Mental Number Line）。该理论认为对数字的加工会自动激活其心 理空间位置, 进而使现实空间中的注意向相应的方向转移, 这样被试在完成数 字大小比较或奇偶判断的任务时才会出现一致或不一致的空间一数字反应编码, 当反应键位置与数字表征在心理数字线上的位置一致时就会易化反应，反之， 则会抑制反应，从而导致了 SNARC 效应的出现。

心理数字线理论提出之后得到了很多实验的支持，其中数字认知领域的另 一个重要现象一一距离效应一直被看作是心理数字线存在的重要证据。该效应 是指对两个数字进行大小比较时，反应时和错误率会随着两个数字之间距离的 增大而减小。这是因为相距较近的数字 (像 7 和 8 ) 位置重叠比较多, 需要更多 的认知资源来分辨两个数字的大小，而相距较远的数字 (如 1 和 8 ) 位置重叠的 程度比较小，区分就更容易一些 [2]［10］。因此，距离效应被看作是数量按 序列表征，即心理数字线存在的证据。

\section{2 极性配对理论}

Proctor 和 Cho 基于极性编码学说提出了极性配对理论, 该理论认为人们可 以对每个刺激和反应都做出 “+” 极和 “-” 极的编码，数字与空间联结的产 生就是因为刺激和反应的极性产生了重叠。具体来讲，在刺激维度上，“大” 意味着 “十”极，“小”意味着 “一”极; 在反应维度上，“右”或 “上”代 表着“十”极，“左”或 “下”代表着 “一”极。当两个维度的极性相同时， 反应速度就会加快; 相反, 当两个维度极性相反时, 反应速度减慢, 所以才会 出现明显的 SNARC 效应 $[11]$ 。

在快速二分类任务 (Speedy Binary Classifi-cation Tasks) 中, 极性编码理论 似乎比心理数字线能够解释得更多。但该理论同样有其局限性，主要表现在两 个方面：首先，极性一致理论强调语义分类的作用，无法解释像数字串对半划 分这样的实验结果; 其次, van Galen 等的实验发现, 当被试的任务是数字大小 
比较时，7-9 岁的儿童均表现出明显的 SNARC 效应，而当被试的任务是数字 奇偶判断时, 只有 9 岁儿童才表现出 SNARC 效应, 对此, van Galen 等认为, 随 着年龄的增长，数字认知逐渐从连续性质发展为分类性质 $[12]$ 。具体而言, 成人和较大年龄的儿童通常使用比较粗粘的言语分类方法将数字序列简单分为 “大” 和 “小” 两类，这可以很好地用两极配对理论来解释; 而年龄较小的儿 童其分类能力尚未成熟、稳定，只能使用有规则的算法程序来比较数字，更多 依赖于空间图像编码的方式, 此时用心理数字线理论来解释更加合理。由此可见， 两极配对理论和心理数字线理论并不是绝对对立的, 更多时候需要二者一起来 为 SNARC 效应的发生做出合理的解释。

\section{3 双路线认知模型}

近年来, Gevers、Caessens 和 Fias (2005) 在 Kornblum 的维度重叠理论的 基础上提出了 SNARC 效应的双路线认知模型 $[13]$ 。该模型认为信息加工有两 条路线，一条是有条件路线，另一条是无条件路线。前者是以任务需求为基础 的慢速路线，即根据任务要求而有意识地控制自己的反应; 而后者是对刺激和 反应之间联系的自动激活（如 SNARC 效应中小数字和左边的联系以及大数字和 右边的联系）。刺激呈现之后，两条路线被平行激活，当任务要求与无条件路 线一致时（即对小数字按左键反应，对大数字按右键反应），两条路线引起的 刺激和反应的联结便是相同的，这就缩短了反应的潜伏期，增加了反应的正确 率; 反之, 当任务要求和无条件路线不一致时, 有条件路线激活的是正确的反应, 而无条件路线激活的是错误的反应，两种反应上的冲突导致反应时变长，正确 率降低 $[14]$ [15］。

双路线认知模型和前两个理论对 SNARC 效应发生时间的解释上存在一定的 差异，前者认为 SNARC 效应发生在晚期的反应选择阶段 [14]，而心理数字线 理论和极性配对理论均强调 SNARC 效应发生在早期的刺激表征阶段 [2 ］［7］。

\section{4 计算理论模型}

为了提供一个解释和预测 SNARC 效应的综合框架, Gevers 等 (2006) 把双 
路线模型的一些基本概念和数字一空间表征特定领域结合起来，提出了计算理 论模型。该模型由上、中、下三层组成：最底层代表心理数字线，包含 “数域” 和 “标准域”，其中数域负责对刺激的数量进行表征和编码; 标准域负责对比 较数字大小时的参照标准进行编码。中间层负责接收来自数域和标准域的处理 结果，并根据任务的要求把这些刺激简单分类。模型的最上层负责反应的选择， 该层共由两个相互联系的节点组成，一个节点负责左侧的反应，另一个节点负 责右侧的反应，如果在反应过程中达到了某个节点的阈限值，这个节点就会被 激活，相应一侧的反应就会发生 $[14]$ 。Notebaert 等（2006）在中间层和最上 层之间增加了 “抽象空间编码层”，认为一个数字被归类为大数或小数后，首 先会激活抽象空间编码层（如 “左” 或 “右”），然后再激活相应的反应。

计算理论模型和极性配对理论都涉及对数字的分类这一中间环节，而心理 数字线理论则强调数字对空间特性的直接激活。此外，计算理论模型还可以解 释以面积 $[16]$ 、长度、亮度、音调以及色调 $[17]$ 为刺激材料的实验所呈现 出的 SNARC 效应，因为这些属于连续量的非符号数量可以通过中间环节进行抽 象分类再映射到心理数字线上，从而出现反应位置与刺激相对大小的反应偏向。

\section{SNARC 效应成因的探索}

\section{1 抽象数量信息和具象数量信息的作用}

抽象的数量信息通常指的是符号数字，如阿拉伯数字、大写的汉语数字以 及各种外文数字等, 而具象的数量信息通常指非符号形式的数量, 如用点数、 木棒数、面积、重量或高度等表示数量大小。胡林成和熊哲宏（2016）在研究 中同时引人了抽象数量材料 (如阿拉伯数字) 和具象数量材料 (如图形的面 积），分别运用面积大小比较和数字大小比较两类任务探讨究竟是哪一种数量 信息导致了 SNARC 效应的出现，结果发现 5 岁的幼儿在数字比较任务中没有出 现 SNARC 效应; 但在面积比较任务中出现了 SNARC 效应，这就表明在 SNARC 效应发生的时序问题上，对具象数量信息的表征在前，而对抽象数量信息的表 征在后，进一步推断出抽象数量信息和具象数量信息都会对 SNARC 效应产生影 
响 [16］。但任杰等 (2013) 在单独使用不同面积的圆进行大小比较时没有发 现 SNARC 效应，而将阿拉伯数字与圆形相结合之后（即在圆的中央根据圆的面 积分别标记 $1 、 4 、 6 、 9$ 四个数字) 再进行面积大小比较时却发现了 SNARC 效应, 这在某种程度上说明抽象数量信息才是引起 SNARC 效应的原因 [18 ]。可见单 纯由具象数量信息是否能引起 SNARC 效应还存在一定的争议。

\section{2 大小信息和顺序信息的作用}

大量研究发现，SNARC 效应普遍存在于符号数字中 [19］［20］，但数字 同时携带大小信息和顺序信息，那么 SNARC 效应究竟是由大小信息引起的还是 由顺序信息引起的呢? 针对这一问题，van Dijck 等提出了对 SNARC 效应中的数 字大小和顺序信息的作用进行分离的想法，结果发现，被试在系列学习数字之 后，系列前面的数字会引起空间注意向左偏移，而系列后面的数字会引起空间 注意向右偏移，说明数字顺序信息在 SNARC 效应中的作用要大于数字本身的 大小信息 $[21]$ 。也有研究采用只携带顺序信息的材料一一字母对顺序信息在 SNARC 效应中的作用进行探索 [22］［23］［24］，结果发现，单纯具有一定 顺序信息的字母也能导致 SNARC 效应的出现 [22］，进一步验证了顺序信息在 SNARC 效应中扮演的重要角色。

鉴于以往对字母加工中的 SNARC 效应都是以字母表为参考系的，王强强等 （2015）创新性地在研究中又引人了一个新的参考系，即不同字母按一定规则 组成的单词。通过启动不同参考系的方法改变字母的顺序从而研究顺序信息对 SNARC 效应的影响。结果发现同样 4 个字母 $\mathrm{C} 、 \mathrm{E} 、 \mathrm{~T} 、 \mathrm{~W}$, 在以字母表为参考系时, $\mathrm{C} 、 \mathrm{E}$ 会引起注意左偏， $\mathrm{T} 、 \mathrm{~W}$ 会引起注意右偏；而以 TWICE 为参考系时， $\mathrm{C} 、 \mathrm{E}$ 会引起注意右偏， T、W 会引起注意左偏 [25］。两种参考系下字母相对位置颠 倒能够引起字母加工中 SNARC 效应的反转, 更加说明顺序信息是导致 SNARC 效应的一个主要因素。

\section{3 具身认知的影响}

具身认知观从一个新的视角去理解 SNARC 效应, 强调认知主体的身体对认 
知活动的影响，这里的身体不仅指脑或者神经机制等身体的解剖学结构，而且 包括宏观上的身体构造和状态、身体的活动方式、身体的物理属性及身体特殊 的感觉一运动通道等方面 [26]［27］。我们下面将从身体状态、主动的身体 运动和被动的身体运动三个方面来阐述具身认知对 SNARC 效应的影响。

\subsection{1 身体状态对 SNARC 效应的影响}

Eerland、Guadalupe 和 Zwaan（2011）为研究身体状态对 SNARC 效应的影 响设计了一个实验，他们让被试在不同的身体姿势下估计埃菲尔铁塔的高度

28]。结果发现，被试身体左倾时对埃菲尔铁塔高度的估计比身体直立和右 倾时估计的明显要小，从而证实身体的物理状态确实会影响人们对数量的表征。

此外, 具身认知观认为, 具身既是认知的, 同时也是嵌人的。大脑嵌人身体, 身体又嵌人环境，从而构成一个完整的认知系统。基于此，张丽等从具身的视 角研究了身体形式和社会环境对 SNARC 效应的影响, 结果发现只有在身体位置 和反应手完全一致的条件下才出现 SNARC 效应，进而说明人们的身体形式及其 所处的社会情境都会对数字认知活动产生影响，大脑、身体和环境共同构成了 一个完整的认知系统。

\subsection{2 主动的身体运动对 SNARC 效应的影响}

大量研究表明，在没有视觉空间表征的情况下，主动的身体运动引起的内 部感受器的激活也会对数字认知产生影响。Loetscher 等 (2008) 的研究发现, 头部的主动转动方向会影响数字认知, 相比于头部向右转动, 向左转时报告小 数字的次数更加频繁，表明积极的头部转动会把注意引向心理数字线空间相对 应的一侧，从而激活这一侧的数字表征。后续研究还发现了垂直方向上的头部 运动对 SNARC 效应的影响, 即相比于头部向上运动, 头部向下运动时报告小数 字的次数更加频繁 [3]。再次证明空间与数字的联合编码不仅是一条心理数字线, 更有可能是一张心理地图 [29］。

主动的身体运动对 SNARC 效应的影响不仅有行为数据的支持，也有来自眼 动数据的证据。Loetscher 等 (2010) 的研究发现，被试眼动的方向可以预测 500 $\mathrm{ms}$ 之后其所随机报告的数字的大小。当眼球向右运动时，被试倾向于报告较大 的数字; 而当眼球向左运动时，被试倾向于报告较小的数字 [30]。据此研究 
者们认为即使是细微的眼动也能引起空间注意的转移, 进而使心理数字线上不 同数字的激活程度存在差异, 导致 SNARC 效应出现。

\subsection{3 被动的身体运动对 SNARC 效应的影响}

虽然很多研究已经证实了主动的身体运动会对 SNARC 效应产生影响, 但同 时也引出一个新的问题：被动的身体运动是否也能引起空间注意的转移呢? 因 为一个头部的主动转动就包含了多个过程, 如预先的头部运动意图、运动指令 的产生和输出、肌肉活动以及脖子和前庭觉系统给出的感觉反馈等，而被动的 身体运动则主要激起来自前庭觉系统的感觉输人而没有其他的过程。

为了证实微弱的前庭觉激活能否同样影响 SNARC 效应, Hartmann 等 (2012) 对此设计了一系列的实验, 并引进了一个可以实现向各个方向 (上、下、左、右、 前、后）被动运动的机器。实验一采用随机数字报告范式检验被动的身体运动 是否会影响 SNARC 效应，结果发现在前后方向上没有发现 SNARC 效应，而在 左右方向和上下方向上均出现了显著的 SNARC 效应。实验二采用数字大小比较 范式同样发现了 SNARC 效应的存在，此外，还发现被试对 “内侧” 数字，即距 离标准数字 5 较近的数字 (4 和 6 ) 的反应时要长于对 “外侧” 数字, 即距离标 准数字 5 较远的数字 $(1 、 2 、 3 、 7 、 8 、 9)$, 也就是出现了距离效应。相比于 极性配对理论, Hartmann 等的这一研究结果更加支持了心理数字线理论, 即数 字并不是简单地以类别来表征 $($ 小数 $=$ 左边, 大数 $=$ 右边 $)$, 而是用公制的空 间维度来表征的 [6]。同时 Hartmann 等的研究最终证实了微弱的前庭觉信息 激活同样是 SNARC 效应的一个重要的影响因素。

\section{SNARC 效应的脑机制}

Dehaene 等 (1996) 记录被试在完成数字大小比较任务时的 ERP 发现, 下 顶叶皮质的激活与用于比较的数字之间的距离成正比，而与数字的呈现方式无 关。据此, Dehaene 等认为距离效应和 SNARC 效应都可以追溯到下顶叶皮质。

与此同时, 认知神经心理学的研究为数字和空间之间的相互联系提供了更 直接的证据。Walsh (2003) 和 Turconi (2006) 的研究都指出顺序、数量、时 间与空间的认知活动激活了相同的顶叶和前额叶皮质区。Dehaene 等 (2003) 
认为, 水平顶内沟 (HIPS) 在数量表征中起着重要作用, 它独立于数字符号, 进行数字处理时会被激活且激活水平随着数量处理的加强而上升; 左侧角回 区（Left Angular Gyrus）是处理言语形式数字的神经基础；双侧后上顶叶系统

( Bilateral Posterior Superior Parietal System) 是心理数字线上空间注意定向的 神经基础，若顶叶损伤，那么空间和数量加工则会出现障碍。

另外, Cutini 等( 2012 )使用功能近红外光谱技术, 在数量表征的核心区域( 双 侧顶内沟 ）和言语数字处理的区域（左侧角回区）都发现了 SNARC 效应的识别 标志，所以数字和空间之间的相互作用，即 SNARC 效应产生于顶叶皮层。实验 研究结果还指出数字一空间相互作用的语义认知核心，不同形式呈现的数字刺 激, 首先进人语义认知核心表征, 进行空间编码, 然后才进行认知加工, 从而 揭示数量表征的内在空间特性，驳乕了 SNARC 效应完全局限于反应选择阶段的 观点 $[31$ 。所以 SNARC 效应的脑机制研究在一定程度上有利于解决 SNARC 效应的理论争议和效应发生的阶段问题。

\section{SNARC 效应研究展望}

综合上述研究可以发现, 近期研究者们很重视对比研究并且已经在 SNARC 效应产生的原因及其与空间注意的关系等方面取得了大量富有价值的研究成果, 但也有一些内容仍存在争议，还需进一步深人探讨。

首先，数字的表征究竟是一个两维的 “心理数字图” 还是一个三维的 “心 理数字空间” 这取决于是否真正存在前后走向的心理数字线，但迄今为止，还 没有实验证据来证明前后方向的 SNARC 效应的存在，究竟是假设不成立，还是 实验设计不够合理都有待进一步检验。

然后, 目前对两位数甚至多位数的 SNARC 效应的研究还比较少, 而且为数 不多的几项研究结果还存在不一致的地方, 如 Dehaene 探讨了两位阿拉伯数字 的SNARC 效应,结果出现了明显的 SNARC 效应, 而 Nuerk、Weger 和 Willmes 2001 ) 以两位数为刺激材料却发现，某些数字能否产生 SNARC 效应受到实验任务的影 响 [32]。所以多位数与一位数在表征方式上可能有着重要的区别，未来的研 究可以在神经机制层面更深人地考察这个问题。 
最后，针对数字同时携带大小信息和顺序信息这个问题，有研究采用只 携带顺序信息的字母为实验材料，发现单纯具有一定顺序信息的字母能导致 SNARC 效应的出现 [22]。另外胡林成和熊哲宏（2016）的研究中引人了具象 数量材料（图形的面积），运用面积大小比较任务进行研究，发现 5 岁的幼儿 就能在面积比较任务中出现 SNARC 效应, 说明单纯的数量信息也能引发 SNARC 效应。既然数量信息和顺序信息同样都能引起 SNARC 效应, 那么二者的加工机 制是否一致呢? 如果不一致的话又分别是什么样子的呢? 相信这会是未来很有 价值的研究课题。

\section{参考文献}

[1] Dehaene S, Dupoux E, Mehler J. Is numerical comparison digital? Analogical and symbolic effects in two-digit number comparison $[\mathrm{J}]$. Journal of Experimental Psychology: Human Perception and Performance, 1990, 16 ( 3 ): 626-641. https://doi.org/10.1037//0096-1523.16.3.626

[2] Dehaene S, Bossini S, Giraux P. The mental representation of parity and number magnitude [ J ] . Journal of Experimental Psychology: General，1993, 122 ( 3 ) : 371-396. https://doi.org/10.1037/0096-3445.122.3.371

[ 3 ] Loetscher T, Schwarz U, Schubiger M, et al. Head turns bias the brain $"$ "s internal random generator [ J ] . Current Biology, 2008, 18 ( 2 ) : 60-62. https://doi.org/10.1016/j.cub.2007.11.015

[4] Hartmann M, Grabherr L, Mast F W . Moving along the mental number line: Interactions between whole-body motion and numerical cognition. [ J ] . Journal of Experimental Psychology Human Perception \& Performance, 2012, 38 ( 6 ) : 1416-1427. https://doi.org/10.1037/a0026706

[ 5 ] Fischer M H, Castel A D, Dodd M D, et al. Perceiving numbers causes spatial shifts of attention $[\mathrm{J}]$. Nature Neuroscience, 2003, 6 ( 6 ) : 555-556. https://doi.org/10.1038/nn1066

[6] Ito Y, Hatta T . Spatial structure of quantitative representation of numbers: 
Evidence from the SNARC effect [ J ] . Memory \& Cognition, 2004, 32 ( 4) : 662-673.https://doi.org/10.3758/BF03195857

[7] Schwarz W, Keus I M . Moving the eyes along the mental number line: comparing SNARC effects with saccadic and manual responses. [ J ] . Perception \& Psychophysics, 2004, 66 ( 4 ) : 651-664.

https://doi.org/10.3758/BF03194909

[ 8 ] Ristic J, Wright A, Kingstone A . The number line effect reflects top-down control [ J ] . Psychonomic Bulletin \& Review, 2006, 13 ( 5 ) : 862-868. https://doi.org/10.3758/BF03194010

[9] 张宇, 雷维娜, 游旭群. 负数的低水平加工引起的空间注意转移一一如果 心理数字线可以延伸至零的左边 $[\mathrm{J}]$. 心理科学, 2010 (4)：819-822.

[ 10 ] Gallistel C R, Gelman R . Preverbal and verbal counting and computation [ J ] . Cognition, 1992, $44(1-2): 43-74$. https://doi.org/10.1016/0010-0277(92)90050-R

[11] Proctor R W, Cho Y S. Polarity correspondence: A general principle for performance of speeded binary classification tasks. [ J ] . Psychological Bulletin, 2006, 132 ( 3 ) : 416-442.

https://doi.org/10.1037/0033-2909.132.3.416

[12] Galen M S V, Reitsma P . Developing access to number magnitude: A study of the SNARC effect in 7- to 9-year-olds [ J ] . Journal of Experimental Child Psychology, 2008, 101 ( 2 ): 99-113. https://doi.org/10.1016/j.jecp.2008.05.001

[13 ] Gevers W, Caessens B, Fias W . Towards a common processing architecture underlying Simon and SNARC effects [ J ] . European Journal of Cognitive Psychology, 2005, 17 ( 5 ) : 659-673.

\section{https://doi.org/10.1080/09541440540000112}

[14] Gevers W, Verguts T, Reynvoet B, et al. Numbers and space: A computational model of the SNARC effect. [ J ] . Journal of Experimental Psychology: Human Perception and Performance, 2006, 32 ( 1 ) : 32-44. 
https://doi.org/10.1037/0096-1523.32.1.32

[15] 张丽, 陈雪梅, 王琦, 等.身体形式和社会环境对 SNARC 效应的影响: 基于具身认知观的理解 $[\mathrm{J}$ ] . 心理学报，2012，44（10）. https://doi.org/10.3724/SP.J.1041.2012.01309

[16] 胡林成, 熊哲宏. 符号数量和非符号数量的空间表征：5 岁儿童的 SNARC 效应和距离效应 $[\mathrm{J}$ ] . 心理科学，2016（2）：364-370.

[ 17 ] Fumarola A,Prpic V,Pos O, et al. Automatic spatial association for luminance[ J ]. Attention Perception \& Psychophysics, 2014, 76 ( 3 ) : 759-765. https://doi.org/10.3758/s13414-013-0614-y

[ 18] 任杰，苏培伟，甄宽. 图形材料加工中的 SNARC 效应：数量信息与顺序 信息的作用 $[\mathrm{J}]$. 心理研究，2013，6（6）：45-50.

[19] 刘超, 买晓琴, 傅小兰.不同注意条件下的空间 -数字反应编码联合效应 $[\mathrm{J}]$. 心理学报, 2004, 36 (6) : 671-680.

[20] Calabria M, Rossetti Y . Interference between number processing and line bisection: a methodology $[\mathrm{J}]$. Neuropsychologia, 2005, 43 ( 5 ) : 779783.https://doi.org/10.1016/j.neuropsychologia.2004.06.027

[21] Van Dijck J P, Abrahamse E L, Majerus S, et al. Spatial Attention Interacts With Serial-Order Retrieval From Verbal Working Memory [ J ] . Psychological Science, 2013, 24 ( 9 ) : 1854-1859.

https://doi.org/10.1177/0956797613479610

[22] Gevers W, Reynvoet B, Fias W . The mental representation of ordinal sequences is spatially organized $[\mathrm{J}]$. Cognition, 2003, 87 ( 3 ) : B87-B95. https://doi.org/10.1016/S0010-0277(02)00234-2

[23 ] Dodd M D, Stigchel S V D, Leghari M A, et al. Attentional SNARC: There's something special about numbers ( let us count the ways ) $[\mathrm{J}]$. Cognition, 2008, 108 ( 3 ) : 810-818. https://doi.org/10.1016/j.cognition.2008.04.006

[24] Previtali P, Hevia M D D, Girelli L . Placing order in space: the SNARC effect in serial learning [ J ] . Experimental Brain Research, 2010, 201 ( 3 ) : 
599-605.https://doi.org/10.1007/s00221-009-2063-3

[25] 王强强，康静梅，兰继军. 顺序信息对注意 SNARC 效应的影响：基于不 同参考系下字母 SNARC 效应的比较研究 [ J ]. 应用心理学, 2015, 21 (4 ): 291-298.

[26] 叶浩生. 具身认知: 认知心理学的新取向 $[\mathrm{J}$ ] . 心理科学进展，2010，18 ( 5 ) : 705-710.

[27 ] 陈雪梅.身体形式和社会环境对 SNARC 效应的影响 [D ]. 西南大学, 2012..

[28 ] Eerland A, Guadalupe T M, Zwaan R A . Leaning to the Left Makes the Eiffel Tower Seem Smaller: Posture-Modulated Estimation [ J ] . Psychological Science, 2011, 22 ( 12 ) : 1511-1514. https://doi.org/10.1177/0956797611420731

[29] Winter B, Matlock B. More is up * and right: Random number generation along two axes $[\mathrm{C}] / /$ Proceedings of the 35th Annual Conference of the Cognitive Science Society. Austin, TX: Cognitive Science Society, 2013.

[ 30 ] Loetscher T, Bockisch C J, Nicholls M E, et al. Eye position predicts what number you have in mind. Current Biology, 2010, 20 ( 6 ) : R264-R265. https://doi.org/10.1016/j.cub.2010.01.015

[31 ] Cutini S, Scarpa F, Scatturin P, et al. Number-Space Interactions in the Human Parietal Cortex : Enlightening the SNARC Effect with Functional NearInfrared Spectroscopy [ J ] . Cerebral Cortex, 2012, 24 ( 2 ) : 444-451. https://doi.org/10.1093/cercor/bhs321

[ 32 ] Nuerk H C, Weger U, Willmes K . Decade breaks in the mental number line? Putting the tens and units back in different bins $[\mathrm{J}]$. Cognition, 2001, 82( 1 ): 25-33. https://doi.org/10.1016/S0010-0277(01)00142-1 